\title{
TRANSFORMATIONS ON DENSITY OPERATORS THAT LEAVE THE HOLEVO BOUND INVARIANT
}

\author{
LAJOS MOLNÁR AND GERGŐ NAGY
}

\begin{abstract}
For a given probability distribution $\lambda_{1}, \ldots, \lambda_{m}$ we determine the structure of all such maps defined on a dense subset of density operators which leave the Holevo bound invariant i.e. which satisfy

$$
S\left(\sum_{k=1}^{m} \lambda_{k} \phi\left(\rho_{k}\right)\right)-\sum_{k=1}^{m} \lambda_{k} S\left(\phi\left(\rho_{k}\right)\right)=S\left(\sum_{k=1}^{m} \lambda_{k} \rho_{k}\right)-\sum_{k=1}^{m} \lambda_{k} S\left(\rho_{k}\right)
$$

for all possible collections $\rho_{1}, \ldots, \rho_{m}$ of density operators.
\end{abstract}

The Holevo bound (or Holevo information) plays a distinguished role in quantum information theory. It provides an upper limit (which can be achieved in certain cases) of the accessible information in a quantum measurement, it gives an upper bound on how well we can infer the value of an unknown random variable based on the knowledge of another one. The definition of this quantity is as follows.

Given a probability distribution $\lambda_{1}, \ldots, \lambda_{m}$, for any collection $\rho_{1}, \ldots, \rho_{m}$ of quantum states, the corresponding Holevo bound is

$$
\chi\left(\rho_{1}, \ldots, \rho_{m}\right)=S\left(\sum_{k=1}^{m} \lambda_{k} \rho_{k}\right)-\sum_{k=1}^{m} \lambda_{k} S\left(\rho_{k}\right),
$$

where $S($.) stands for the usual von Neumann entropy, i.e., $S(\rho)=-\operatorname{tr} \rho \log \rho$ for any state $\rho$. Observe that by the concavity of the von Neumann entropy, $\chi$ is always nonnegative. We recall that the Holevo bound is the keystone in the proof of many results of quantum information theory, more details can be found in any book on quantum information science, here we refer only to the classic monograph [5].

Motivated by Wigner's fundamental theorem on quantum mechanical symmetry transformations which describes the structure of all maps on the space of pure states that preserve the quantity of transition probability, in this paper we determine those state-transformations which leave the Holevo bound invariant. We recall that in the paper [4] Molnár and Timmermann determined those bijective maps of the space $\mathcal{S}(H)$ of all density operators on a finite dimensional Hilbert space $H$ which preserve the Jensen-Shannon

The authors were supported by the "Lendület" Program (LP2012-46/2012) of the Hungarian Academy of Sciences. The first and second author were supported by the Hungarian Scientific Research Fund (OTKA) Reg.No. K81166 NK81402. The second author was supported by the TÁMOP-4.2.2/B-10/1-2010-0024 project co-financed by the European Union and the European Social Fund. 
divergence $D_{J S}(. \|$.$) . Originally, that quantity was defined as a symmetrized$ version of the relative entropy but in the present setting we prefer writing it in the form

$$
D_{J S}(\rho \| \sigma)=S\left(\frac{\rho+\sigma}{2}\right)-\frac{1}{2} S(\rho)-\frac{1}{2} S(\sigma) .
$$

It is apparent that $D_{J S}$ can be viewed as a particular Holevo bound. We point out that $\sqrt{D_{J S}}$ is conjectured to be a true metric on the space of all density operators. That would be a really remarkable property, unfortunately proof is given only in the 2-dimensional case [1].

Below we describe the structure of all transformations defined on a "large part" of the space of density operators which preserve the Holevo bound, i.e. we determine all those "symmetries" of the state-space which leave the important quantity of the Holevo bound invariant. Observe that this result significantly generalizes the former theorem [4] in different directions: The quantity that we consider here reduces to the Jensen-Shannon divergence in a particular case, the transformations under consideration are not assumed to be surjective and not assumed to be defined on the whole space $\mathcal{S}(H)$ either. This latter generalization is important since in particular it allows us to consider transformations defined only on the set of all non-singular density operators, a subset of $\mathcal{S}(H)$ that plays an important role when differential geometrical tools are to be used in quantum information theory or quantum statistics.

Turning to our result, it is apparent that for any unitary or antiunitary operator $U: H \rightarrow H$, the transformation $\rho \mapsto U \rho U^{*}$ on $\mathcal{S}(H)$ preserves the Holevo bound i.e. we have

$$
\chi\left(U \rho_{1} U^{*}, \ldots, U \rho_{m} U^{*}\right)=\chi\left(\rho_{1}, \ldots, \rho_{m}\right)
$$

for any collection $\rho_{1}, \ldots, \rho_{m}$ of quantum states. The theorem below asserts that the converse statement is also true, every state-transformation that leaves the Holevo bound invariant is induced by a unitary or antiunitary operator.

More precisely, we have the following result. We point out that whenever we use topological or metrical notions they always refer to the topology or the metric induced by the trace norm $\|\cdot\|_{1}$.

Theorem 1. Let $H$ be a complex Hilbert space with $n=\operatorname{dim} H<\infty$. Let a probability distribution $\lambda_{1}, \ldots, \lambda_{m}$ be given, all $\lambda_{k}$ 's are positive real numbers and their sum is 1 . Assume that $\phi: \mathcal{M} \rightarrow \mathcal{S}(H)$ is a transformation defined on a dense subset $\mathcal{M}$ of $\mathcal{S}(H)$ with the property that

$$
S\left(\sum_{k=1}^{m} \lambda_{k} \phi\left(\rho_{k}\right)\right)-\sum_{k=1}^{m} \lambda_{k} S\left(\phi\left(\rho_{k}\right)\right)=S\left(\sum_{k=1}^{m} \lambda_{k} \rho_{k}\right)-\sum_{k=1}^{m} \lambda_{k} S\left(\rho_{k}\right)
$$

holds for all collections $\rho_{1}, \ldots, \rho_{m}$ of density operators in $\mathcal{M}$. Then there is a unitary or antiunitary operator $U$ on $H$ such that

$$
\phi(\rho)=U \rho U^{*} \quad(\rho \in \mathcal{M}) .
$$


Proof. We assume that

$$
S\left(\sum_{k=1}^{m} \lambda_{k} \phi\left(\rho_{k}\right)\right)-\sum_{k=1}^{m} \lambda_{k} S\left(\phi\left(\rho_{k}\right)\right)=S\left(\sum_{k=1}^{m} \lambda_{k} \rho_{k}\right)-\sum_{k=1}^{m} \lambda_{k} S\left(\rho_{k}\right)
$$

holds for all possible collections $\rho_{1}, \ldots, \rho_{m}$ of density operators. Choosing $\rho_{2}=\rho_{3}=\ldots=\rho_{m}$ and defining $\rho_{0}=\rho_{2}=\rho_{3}=\ldots=\rho_{m}$ and $\lambda_{0}=$ $\lambda_{2}+\ldots+\lambda_{m}=1-\lambda_{1}$ we see that $\phi$ satisfies

$$
\left.S\left(\sum_{k=0}^{1} \lambda_{k} \phi\left(\rho_{k}\right)\right)\right)-\sum_{k=0}^{1} \lambda_{k} S\left(\phi\left(\rho_{k}\right)\right)=S\left(\sum_{k=0}^{1} \lambda_{k} \rho_{k}\right)-\sum_{k=0}^{1} \lambda_{k} S\left(\rho_{k}\right) .
$$

Hence the original problem where we consider a given probability distribution and collections of density operators all consisting of $m$ members can be reduced to the question relating to collections with only two elements.

Accordingly, for a given number $\lambda \in] 0,1[$ and for any pair $\rho, \sigma \in \mathcal{S}(H)$ of density operators we define

$$
\chi_{\lambda}(\rho, \sigma)=S(\lambda \rho+(1-\lambda) \sigma)-\lambda S(\rho)-(1-\lambda) S(\sigma)
$$

and we assume that $\phi: \mathcal{M} \rightarrow \mathcal{S}(H)$ is a map which satisfies

$$
\chi_{\lambda}(\phi(\rho), \phi(\sigma))=\chi_{\lambda}(\rho, \sigma)
$$

for all $\rho, \sigma \in \mathcal{M}$.

The first important step of the proof follows where we extend $\phi$ onto the whole set $\mathcal{S}(H)$. To do this we need a lower and an upper bound for $\chi_{\lambda}(.,$. in terms of the trace norm $\|\cdot\|_{1}$. To obtain the upper bound, let $\eta:[0,1] \rightarrow \mathbb{R}$ be the function defined by

$$
\eta(x)= \begin{cases}-x \log x, & x \in] 0,1] \\ 0, & x=0\end{cases}
$$

Pick operators $\rho, \sigma \in S(H)$ satisfying $\|\rho-\sigma\|_{1} \leq 1 / e$. By Fannes' inequality (cf., e.g. [5, Theorem 11.6]) we have

$$
|S(\rho)-S(\sigma)| \leq\|\rho-\sigma\|_{1} \log n+\eta\left(\|\rho-\sigma\|_{1}\right)
$$

and this yields that

$$
\begin{gathered}
\chi_{\lambda}(\rho, \sigma) \leq \\
\leq \lambda|S(\lambda \rho+(1-\lambda) \sigma)-S(\rho)|+(1-\lambda)|S(\lambda \rho+(1-\lambda) \sigma)-S(\sigma)| \\
\leq(2 \lambda(1-\lambda) \log n)\|\rho-\sigma\|_{1}+\lambda \eta\left((1-\lambda)\|\rho-\sigma\|_{1}\right)+(1-\lambda) \eta\left(\lambda\|\rho-\sigma\|_{1}\right) .
\end{gathered}
$$

As for the lower bound, let $\rho, \sigma \in \mathcal{S}(H)$. We denote by $S(. \|$.) the quantum relative entropy (cf., [5]). On the one hand, we learn from [2, Theorem III.1] that

$$
S(\rho \| \sigma) \geq \frac{\|\rho-\sigma\|_{1}^{2} \log e}{2} .
$$

On the other hand, according to [1], simple computation shows that

$$
\chi_{\lambda}(\rho, \sigma)=\lambda S(\rho \| \lambda \rho+(1-\lambda) \sigma)+(1-\lambda) S(\sigma \| \lambda \rho+(1-\lambda) \sigma) .
$$


Therefore we derive

$$
\lambda(1-\lambda) \log e\|\rho-\sigma\|_{1}^{2} \leq \chi_{\lambda}(\rho, \sigma) .
$$

Now, observe that the inequality (1) implies that for small enough $\|\rho-\sigma\|_{1}$, the quantity $\chi_{\lambda}(\phi(\rho), \phi(\sigma))=\chi_{\lambda}(\rho, \sigma)$ is small and next, (2) applied for $\phi(\rho)$ and $\phi(\sigma)$ tells us that then $\|\phi(\rho)-\phi(\sigma)\|_{1}$ is also small. It follows that $\phi$ is uniformly continuous with respect to the metric induced by $\|\cdot\|_{1}$. We have assumed that $\mathcal{M}$ is dense in $\mathcal{S}(H)$ which is a complete metric space and hence using an elementary result from classical analysis we deduce that $\phi$ has a unique uniformly continuous extension $\tilde{\phi}: \mathcal{S}(H) \rightarrow \mathcal{S}(H)$.

In what follows we show that $\tilde{\phi}$ is of the form stated in the theorem. To this end, first we claim that $\tilde{\phi}$ also preserves $\chi_{\lambda}(.,$.$) . Indeed, as \tilde{\phi}$ preserves this quantity on the dense set $\mathcal{M}$ and $\chi_{\lambda}(.,$.$) is easily seen to be a continuous$ function of its variables, by the continuity of $\tilde{\phi}$ it follows that

$$
\chi_{\lambda}(\tilde{\phi}(\rho), \tilde{\phi}(\sigma))=\chi_{\lambda}(\rho, \sigma)
$$

holds for all $\rho, \sigma \in \mathcal{S}(H)$. We next show that $\tilde{\phi}$ preserves the orthogonality meaning that for any $\rho, \sigma \in \mathcal{S}(H)$ we have

$$
\tilde{\phi}(\rho) \tilde{\phi}(\sigma)=0 \Longleftrightarrow \rho \sigma=0 .
$$

Let $\rho, \sigma \in \mathcal{S}(H)$. Hopefully it causes no serious confusion if, as usual, we denote by $H($.$) the classical Shannon entropy of probability distributions.$ The statement [5, Theorem 11.10] tells us that

$$
S(\lambda \rho+(1-\lambda) \sigma) \leq \lambda S(\rho)+(1-\lambda) S(\sigma)+H(\{\lambda, 1-\lambda\})
$$

and here equality occurs if and only if $\rho \sigma=0$. Therefore we obtain that $\rho \sigma=0$ holds exactly when $\chi_{\lambda}(\rho, \sigma)=H(\{\lambda, 1-\lambda\})$. Referring to (3) this yields immediately that $\tilde{\phi}$ preserves the orthogonality. Denote by $\mathcal{P}_{1}(H)$ the set of all rank-one projections on $H$. Observe that the elements of $\mathcal{P}_{1}(H)$ can be characterized as those operators in $\mathcal{S}(H)$ which are in a set of $n$ pairwise orthogonal density operators on $H$. Now we get that $\phi\left(\mathcal{P}_{1}(H)\right) \subset \mathcal{P}_{1}(H)$.

Let $p, q \in \mathcal{P}_{1}(H)$ be different projections. We are going to derive a formula for $\chi_{\lambda}(p, q)$. In order to do this, first observe that the von Neumann entropy of any element of $\mathcal{P}_{1}(H)$ is 0 , thus we have $\chi_{\lambda}(p, q)=S(\lambda p+(1-\lambda) q)$. Now let $x$, resp. $y$ be a unit vector in the range of $p$, resp. $q$. If we restrict $\lambda p+(1-\lambda) q$ to its range, then the matrix of the restriction with respect to the basis $\{x, y\}$ is

$$
\left(\begin{array}{cc}
\lambda & \lambda\langle y, x\rangle \\
(1-\lambda)\langle x, y\rangle & 1-\lambda
\end{array}\right) .
$$

Using the formula $\operatorname{tr} p q=|\langle x, y\rangle|^{2}$, we deduce that the spectrum of this matrix is

$$
\left\{\frac{1+\sqrt{1+4 \lambda(1-\lambda)(\operatorname{tr} p q-1)}}{2}, \frac{1-\sqrt{1+4 \lambda(1-\lambda)(\operatorname{tr} p q-1)}}{2}\right\} .
$$


Since the nonzero eigenvalues of $\lambda p+(1-\lambda) q$ are the elements of the latter set, this implies

$$
\chi_{\lambda}(p, q)=1-\frac{(1+a) \log (1+a)+(1-a) \log (1-a)}{2},
$$

where $a=\sqrt{1+4 \lambda(1-\lambda)(\operatorname{tr} p q-1)}$. Consider the function $f:[0,1[\rightarrow \mathbb{R}$ defined by

$$
f(t)=1-\frac{(1+t) \log (1+t)+(1-t) \log (1-t)}{2} \quad(t \in[0,1[) .
$$

By differentiation we obtain that $f$ is strictly monotone and hence injective. Therefore, it follows that the function $g:[0,1[\rightarrow \mathbb{R}$ given by

$$
g(t)=f(\sqrt{1+4 \lambda(1-\lambda)(t-1)}) \quad(t \in[0,1[)
$$

is also injective. Now we can rewrite (4) in the form

$$
\chi_{\lambda}(p, q)=g(\operatorname{tr} p q) .
$$

By (3) we have $g(\operatorname{tr} \tilde{\phi}(p) \tilde{\phi}(q))=g(\operatorname{tr} p q)$ which yields that

$$
\operatorname{tr} \tilde{\phi}(p) \tilde{\phi}(q)=\operatorname{tr} p q .
$$

Since this equality holds also for equal projections $p, q$ we have that the map $\left.\tilde{\phi}\right|_{\mathcal{P}_{1}(H)}: \mathcal{P}_{1}(H) \rightarrow \mathcal{P}_{1}(H)$ preserves the transition probability (the trace of the product) between the elements of $\mathcal{P}_{1}(H)$. Applying a version of Wigner's theorem (cf., [3, Theorem 2.1.4.]), we conclude that there is a unitary or antiunitary operator $U$ on $H$ such that

$$
\tilde{\phi}(p)=U p U^{*} \quad\left(p \in \mathcal{P}_{1}(H)\right) .
$$

What remains to verify is that the above equality holds not only for rankone projections but for all density operators, too. As already mentioned, in [4] Molnár and Timmermann have described the structure of those bijective transformations on $\mathcal{S}(H)$ which preserve the Jensen-Shannon divergence $D_{J S}(. \|$.$) . As appeared in the introduction we have$

$$
D_{J S}(\rho \| \sigma)=S\left(\frac{\rho+\sigma}{2}\right)-\frac{1}{2} S(\rho)-\frac{1}{2} S(\sigma)=\chi_{\frac{1}{2}}(\rho, \sigma) \quad(\rho, \sigma \in \mathcal{S}(H)) .
$$

In the proof in [4] a several page long and very non-trivial argument was presented to show that given a transformation that preserves the JensenShannon divergence and implemented by a unitary or antiunitary operator on the set of all rank-one projections (meaning that it is of a form similar to $(5))$ is necessarily implemented by that unitary or antiunitary operator on the whole space $\mathcal{S}(H)$. With some work one can check that, with slight modifications, the argument presented in [4] works in our present case as well. In that way one can achieve the same conclusion and verify that $\tilde{\phi}(\rho)=$ $U \rho U^{*}$ holds for all $\rho \in \mathcal{S}(H)$. This completes the proof of Theorem 1 . 


\section{REFERENCES}

1. J. Briët, P. Harremoës, Properties of classical and quantum Jensen-Shannon divergence, Phys. Rev. A, 79 (2009), 052311.

2. H. Klauck, A. Nayak, A. Ta-Shma and D. Zuckerman, Interaction in quantum communication, IEEE Transactions on Information Theory, 53 (2007) 1970-1982.

3. L. Molnár, Selected Preserver Problems on Algebraic Structures of Linear Operators and on Function Spaces, Lecture Notes in Mathematics, Vol. 1895, Springer, 2007.

4. L. Molnár and W. Timmermann, Maps on quantum states preserving the JensenShannon divergence, J. Phys. A: Math. Theor. 42 (2009), 015301.

5. M. Nielsen and I. Chuang, Quantum Computation and Quantum Information, Cambridge University Press, 2000.

MTA-DE "Lendület" Functional Analysis Research Group, Institute of Mathematics, University of Debrecen, H-4010 Debrecen, P.O. Box 12, HunGARY

E-mail address: molnarl@science.unideb.hu

URL: http://www.math. unideb.hu/ molnarl/

E-mail address: nagyg@science.unideb.hu 